\title{
L'agence national pour la promotion des investissements et la promotion de l'Etat de droit : cadre juridique et défis actuels
}

\author{
Par William Katembo Kassilam*
}

\section{Résumé}

Cet article est focalisé sur les défis du secteur des investissements au regard des mécanismes juridiques pour la promotion des investissements en RDC. L'étude présente l'organisation, le fonctionnement et les missions de l'ANAPI qu'est une institution nationale pour appuyer le gouvernement en ce qui concerne la promotion des investissements. Cette institution est créée et organisée par la Loi $n^{\circ}$ 004/2002du 21 février 02 portant code des investissements en République Démocratique du Congo qui détermine les modalités de fonctionnement de l'ANAPI et lui assigne la mission de veiller à ce que l'action du gouvernement puisse promouvoir les investissements pour le développement de la nation. Elle est Organisée aux tours de son Conseil d'administration; du Conseil d'agrément; de la Direction Générale; et du Collège des commissaires aux comptes et accompagne les investisseurs et veille au bon usage de l'aide publique disponible pour appuyer les projets d'investissement viables. Au regards des mécanismes de son fonctionnement et des modalités pour l'octroi des fonds publiques aux investisseurs pour appuyer leurs projets d'investissement, cette article démontre la nécessité du renforcement des mécanismes de contrôle et de suivis en amont tout comme en aval pour que l'appui ou les fonds octroyés aux investisseurs puissent être convenablement utilisés et contribuer au développement du pays.

\begin{abstract}
This article focuses on the challenges of the investment sector with respect to the legal mechanisms for investment promotion in the DRC. The study presents the organization, functioning and missions of ANAPI which is a national institution to support the government in the promotion of investments. This institution is created and organized by the Law $\mathrm{n}^{\circ}$ 004/2002 of February 21, 2002 on the code of investments in the Democratic Republic of Congo which determines the modalities of functioning of the ANAPI and assigns to it the mission to see to it that the action of the government can promote the investments for the development of the nation. It is organized around its Board of Directors; the Approval Council; the General Management; and the College of Auditors and accompanies investors and ensures the proper use of public aid available to support viable investment projects. With regard to the mechanisms of its functioning and the modalities for the granting of
\end{abstract}

* Licencié en droit de l’Université de Kinshasa. 
public funds to investors to support their investment projects, this article demonstrates the need to strengthen the control and monitoring mechanisms upstream as well as downstream so that the support or funds granted to investors can be properly used and contribute to the development of the country.

\section{Introduction}

L'Agence Nationale pour les promotions des Investissements, en sigle " ANAPI 》 a été instituée aux termes des dispositions de l'article 4 de la Loi n ${ }^{\circ}$ 004/2002du 21 février 02 portant code des investissements en République Démocratique du Congo. Elle est un établissement public à caractère technique doté de la personnalité juridique. Elle est l'organe Conseil du Gouvernement Central et des Gouvernements Provinciaux en matière de promotion des investissements et de l'amélioration du climat des affaires, placée sous la tutelle du Ministre ayant le Plan dans ses attributions. Les activités promotionnelles de l'ANAPI touchent tous les secteurs d'activités économiques. Agriculture, Agro-industrie, Elevage, Pêche, Industries manufacturières, Hôtellerie et restauration, Bâtiments et travaux publics, Logements sociaux, Bois et foresterie, Transports aérien, routier, maritime, fluvial, Télécommunications, Technologies de l'information Energie, Textile, Services divers, Mines, Banques et assurances. S'agissant du volet agrément ou admission des projets aux avantages du Code des Investissements, les projets relevant des secteurs des mines, banques et assurances, ne sont pas éligibles et par conséquent, l'ANAPI intervient uniquement pour émettre ses avis techniques.

\section{A. Mission et fonctionnement de l'ANAPI}

Conformément aux textes qui la régissent, à savoir la Loi nº 004/2002 du 21 février 2002 portant Code des Investissements, et le Décret du Premier Ministre n 09/33 du 08 août 2009 portant statuts, organisation et fonctionnement de l'ANAPI, les missions assignées à cette Agence sont les suivantes : Promotion de l'image positive de la République Démocratique du Congo;Promotion des opportunités spécifiques d'investissement; Plaidoyer en vue de l'amélioration du climat des affaires au pays (after-care); Accompagnement administratif des investisseurs qui décident d'établir ou d'étendre leurs activités économiques sur le territoire national ${ }^{1}$.

L'ANAPI est chargée notamment d'assurer en permanence un plaidoyer en vue de l'améliorationdu climat des investissements et de jouer, en cette matière,le rôle de conseil $\mathrm{du}$ Gouvernement central et desGouvernements provinciaux; de travailler à la promotion d'une image positive de laRépublique Démocratique du Congo comme paysd'investissements et d'opportunités pour les investisseurs; d'identifier et de promouvoir, auprès des

1 Article 3, Décret $n^{\circ}$ 09/33 du 08 août 2009 portant statuts, organisation et fonctionnement de l'Agence pour la

Promotion des Investissements, en sigle « ANAPI ». 
investisseursnationaux et internationaux, les opportunités spécifiquesd'investissement en République Démocratique du Congo;d'assurer, aux investisseurs qui décident d'établir ou d'étendreleurs activités économiques sur le territoire congolais, unaccompagnement qui facilite et accélère les procéduresadministratives nécessaires à la réalisation desinvestissements et à la création d'entreprises dans lesmeilleures conditions de délai et de transparence.

La mission de plaidoyer comprend notamment la mise en œuvre, en collaboration avec le Gouvernementcentral et les Gouvernements provinciaux, les entreprises etautres services publics ainsi que les partenaires, dans le cadred'un dialogue entre les secteurs public et privé, des voies etmoyens visant l'élimination des barrières ou tracasseriesadministratives qui touchent aux opérations de création,d'extension et/ou de modernisation des entreprises;L'animation et le suivi des groupes de travail réunissant lesexperts des secteurs public et privé, et axés sur les prioritésd'amélioration du climat des investissements, telles qu'établiessoit par le Gouvernement, les entreprises et les partenaires, soitencore sur base des résultats des sondages obtenus desinvestisseurs existants ou potentiels;- La conduite des études et la formulation de toutes suggestionsutiles soit en vue d'une meilleure application du Code desinvestissements, soit en vue de l'amélioration des incitations denature à promouvoir les investissements, soit encore pour uneamélioration, dans des diverses régions économiques du pays,des conditions d'accueil des investissements nationaux ouétrangers;L'organisation d'un service médiateur pour les investisseurs envue de leur assurer davantage de compétitivité en s'impliquant demanière concrète dans les actions visant l'élimination desbarrières à leur établissement et à leur développement;- La tenue d'une table ronde périodique entre le Gouvernement etles investisseurs;- L'avis motivé de l'ANAPI sur tout projet ou proposition de textelégislatif ou réglementaire susceptible d'entraîner unemodification du climat des investissements en RépubliqueDémocratique du Congo, y compris les dispositions de naturefiscale ou douanière;- L'initiative de proposer aux autorités compétentes des nouvellesdispositions législatives ou réglementaires qui touchent àl'amélioration du climat des investissements;- La participation, en étroite collaboration avec les ministèresconcernés, à la négociation des traités concernant la protectiondes investissements, des traités de libre-échange ainsi que desconventions de prévention de double imposition.

La mission d'accompagnement administratif des investisseurs quidécident d'établir ou d'étendre leurs activités économiques sur leterritoire national comprend notamment:- L'organisation, pour les opérations et procédures visées àl'article 8 ci-dessous, d'un service de guichet unique doté d'unmanuel opérationnel et animé par des agents de l'Etat déléguéspar leurs ministères ou services et disposant de pouvoirs dedécision nécessaires à l'exercice de leurs fonctions;- La réception et l'instruction des projets d'investissement àagréer dans le cadre du Code des investissements et des projetsd'investissements régis par des Lois particulières, en vue dedécider de l'agrément de ceux qui relèvent du Code desinvestissements, ou d'émettre des avis techniques sur les autres;- La surveillance des engagements souscrits par les promoteursdes investissements agréés au bénéfice des avantages du Codedes inves- 
tissements et, en cas de manquement, la possibilité deproposer à l'autorité de tutelle ou aux autorités compétentes,avec des avis motivés, des sanctions à prendre.

Les ressources de l'ANAPI sont constituées des frais de dépôt des dossiers de demande d'agrément desprojets d'investissement fixés par Arrêté conjoint des Ministresayant le Plan et les Finances dans leurs attributions;- des produits de vente des documents ou autres services fournispar l'Agence;- d'une quotité des recettes provenant de la taxe de promotion del'industrie, fixée par Arrêté conjoint des Ministres ayantl'Industrie, le Plan et les Finances dans leurs attributions, aprèsavis du Fonds de Promotion de l'Industrie;- d'une subvention allouée dans le cadre du budget de l'Etat sousforme de dotation;- Eventuellement du solde budgétaire de l'exercice antérieur;- de la contribution des partenaires bi ou multilatéraux;des subventions, dons, legs et libéralités d'origine interne ouexterne dûment acceptés par le Conseil d'administration ${ }^{2}$.

\section{B. L'organisation de l'ANAPI}

Les organes de l'ANAPI sont le Conseil d'administration; le Conseil d'agrément; la Direction Générale; et le Collège des commissaires aux comptes ${ }^{3}$. Le Conseil d'administration définit les orientations stratégiques de l'ANAPI. Il a les pouvoirs les plus étendus pour poser tous les actes d'administration en rapport avec l'objet de l'ANAPI.A ce titre, le Conseil d'administration:- Valide le plan stratégique de l' ANAPI et veille à sa conformité avec les objectifs de développement du Gouvernement;- Valide le plan d'actions annuel, le budget ainsi que les états financiers de fin de l'exercice;- Veille à la mise en place du plan stratégique ainsi qu'à la réalisation des objectifs fixés;- Décide des opérations d'acquisition, de vente ou de prise des participations, des transactions ou des cessions;- Assure le suivi de la performance de la gestion de l'ANAPI;- Décide, sur recommandation de la Direction Générale, del'allocation des primes de performance;- Approuve les accords de financement négociés par la DirectionGénérale avec les bailleurs de fonds et d'autres parties prenantesen vue de l'obtention de ressources supplémentaires pourl'accomplissement des missions de l'ANAPI.Le Conseil d'administration délègue à la Direction Générale tousles pouvoirs nécessaires pour lui permettre d'assurer la gestioncourante de l'ANAPI. Il détermine les directives de cette gestion eten surveille l'exécution.

Le Conseil d'administration de l'ANAPI est constitué de cinqmembres.Outre le Directeur Général, le Conseil d'administration comprendun délégué du Ministère du Plan, un délégué du Ministère desFinances et deux représentants du secteur privé proposés parleurs corporations d'origine, tous nommés sur proposition duMinistre ayant le Plan dans ses attributions, par Ordonnance duPrésident de la République, délibérée en Conseil des Mi-

2 Article 14, Décret $n^{\circ}$ 09/33 du 08 août 2009 portant statuts, organisation et fonctionnement de l'Agence pour la

Promotion des Investissements, en sigle « ANAPI ».

3 Article 15, Décret n 09/33 du 08 août 2009. 
nistres.Le Conseil d'administration est présidé par un représentant dusecteur privé. Il est nommé par le Président de la République surproposition du Ministre ayant le Plan dans ses attributions.Le mandat des membres du Conseil d'administration est de cinqans, renouvelable une fois.Les membres du Conseil d'administration ont droit à un jeton deprésence dont le montant est fixé par le Décret du PremierMinistre délibéré en Conseil des Ministres sur proposition duMinistre ayant le Plan dans ses attributions.Peuvent participer aux réunions du Conseil d'administration, enqualité d'observateurs, sans voix délibérative et suivant lesmodalités arrêtées dans le Règlement intérieur, les représentantsdu secteur public et du secteur privé.

Le Conseil d'agrément est l'organe chargé de statuer sur lesdemandes d'agrément des projets d'investissement éligibles auCode des investissements et d'émettre des avis techniques sur lesprojets d'investissement régis par des Lois particulières.Il est constitué de membres permanents et non permanents. Sontmembres permanents :- Un délégué du cabinet du Président de la République;- Un délégué du cabinet du Premier Ministre;Un délégué du ministère ayant le Plan dans ses attributions;- Un délégué du ministère ayant les Finances dans sesattributions;- Un délégué du ministère ayant le Budget dans ses attributions;- Un délégué du ministère ayant l'Economie dans ses attributions;- Un délégué du ministère ayant l'Environnement dans sesattributions;- Un délégué du ministère ayant l'Industrie dans ses attributions;- Un délégué du ministère ayant le Travail et la PrévoyanceSociale dans ses attributions;- Un délégué de l'Office de Douane et Accises « OFIDA »;- Un délégué de la Direction Générale des Impôts « DG! »;- Un délégué de la Direction Générale des RecettesAdministratives, Judiciaires, Domaniales et de Participation «DGRAD »;- Un délégué du Fonds de Promotion de l'Industrie «FPI »;- Le Directeur Général de l'ANAPI;- Le Directeur Général Adjoint de l'ANAPI.Sont membres non permanents les délégués des ministèresconcernés par les projets d'investissement dont l'ANAPI est saisieet qui sont invités par le Président du Conseil d'agrément, surproposition de la Direction Générale.Chaque membre permanent est pourvu d'un suppléant qui leremplace d'office en cas d'absence ou d'empêchement.Les membres permanents du Conseil d'agrément sont désignéspar leurs services ou organismes respectifs, étant entendu que lamême personne ne peut siéger à la fois au Conseild'administration et au Conseil d'agrément. Ils sont nommés parun Arrêté du Ministre ayant le Plan dans ses attributions.

Le Conseil d'agrément se réunit sur convocation de son Président, en session ordinaire, deux fois par mois et, en sessionextraordinaire, chaque fois que l'intérêt de l'ANAPI l'exige oulorsque la demande en a été faite par écrit, soit par la moitié deses membres, soit par l'autorité de tutelle.En cas d'absence ou d'empêchement du Président du Conseild'agrément, le délégué du Ministère des Finances assure d'officeson intérim.Les convocations sont adressées à chaque membre permanentounon permanent huit jours francs au moins avant la date de latenue de la réunion.Le Conseil d'agrément dispose d'un secrétariat permanent assurépar deux agents de l'ANAPI.Un Règlement intérieur adopté par le Conseil d'agrément etapprouvé par le Ministre ayant le Plan dans ses attributions endétermine les règles de fonctionnement. 
La Direction Générale est l'organe de gestion courante de l'ANAPI. Elle applique les décisions du Conseil d'administration et veille àl'exécution des décisions en matière de promotion desinvestissements et d'agrément des projets aux avantages duCode des investissements.Elle veille au fonctionnement efficace et harmonieux des servicesadministratifs et techniques de l'ANAPI.A ce titre, elle est chargée notamment:- De préparer les orientations stratégiques de l'ANAPI, le pland'action annuel et le budget annuel à soumettre au Conseild'administration pour validation;- D'exécuter, d'une manière efficace et sous le contrôle du Conseild'administration, le plan d'actions annuels et le budget annuelapprouvés;- De coordonner et superviser les services administratifs ettechniques de l'ANAPI, conformément aux Lois et règlements envigueur;- De gérer le personnel, les ressources financières ainsi que lepatrimoine de l'ANAPI;- D'analyser et évaluer, dans les délais impartis, les projetsd'investissement éligibles aux avantages du Code desinvestissements ou régis par des Lois particulières, et dontl'ANAPI est saisie;- De soumettre au Conseil d'agrément, dans les délais impartis,les rapports d'analyse et d'évaluation des projets d'investissementayant fait l'objet d'études techniques;- D'assurer l'exécution, dans le délai légal, des Arrêtésinterministériels d'agrément des projets d'investissement, denotifier les lettres de mise en demeure ainsi que les Arrêtésinterministériels de retrait d'agrément;- De suivre et évaluer l'exécution des engagements souscrits parles promoteurs des investissements agréés et d'en faire rapportau Conseil d'agrément;- De constituer une banque de données en matière depotentialités en investissements en République Démocratique duCongo, de manière générale, et par province, de manièrespécifique;- D'établir les rapports trimestriels et annuels d'activités et desrésultats obtenus, à la demande du Conseil d'administration;- D'étudier toutes les questions en rapport avec l'application du Code des investissements ou toutes questions se rattachant directement ou indirectement à l'objet social de l' ANAPI;La Direction Générale représente l'ANAPI vis-à-vis des tiers. Elle agit en toute circonstance au nom de l'agence et dispose du pouvoir d'ester en justice, tant en demande qu'en défense, poursuites et diligences du Directeur Général.

\section{Les défis actuels de l'ANAPI pour la promotion des investissements en RDC}

La RDC a adopté des mesures d'admission des investissements étrangers incitatives en faisant le choix d'un régime d'agrément. L'idée d'admettre les investissements par un agrément au préalable en RDC remonte aux anciens codes des investissements. Dans le but d'encourager les promoteurs étrangers, privés et publics, à investir dans des activités de nature à contribuer au développement économique et social du pays, le code de 1986 établissait trois régimes privilégiés, à savoir : le régime général, le régime conventionnel et le régime de zone franche à vocation industrielle (ZOFI). Le régime de la zone franche concernait exclusivement les industries lourdes, appartenant aux nationaux et aux expatriés, installées autour du barrage d'Inga et à Kinshasa en vue de l'extraction et de la transfor- 
mation des matières localement avant leur exportation ${ }^{4}$. Ces régimes s'appliquaient aussi bien aux investissements créant des entreprises qu'aux investissements dans des entreprises existantes. Les entreprises bénéficiaires du code pouvaient être aussi bien des entreprises individuelles que des sociétés constituées selon les différentes formes légalement reconnues ${ }^{5}$.

Cette évolution n'est pas allée sans soulever de grandes difficultés pratiques tenant à l'application dudit code. C'est ainsi qu'en 2002 le gouvernement congolais a opté pour un autre système au détriment du précédent. Pour comprendre la démarche du législateur congolais, il nous semble préférable de se référer à l'esprit même de la loi n 004/2002 du 21 février 2002 portant code des investissements, actuellement en vigueur. L'objectif pour le législateur est de créer un cadre juridique propice et attractif à même de s'appliquer à l'ensemble des avantages accordés aux investisseurs et investissements étrangers admis en RDC. De ce fait, le législateur établit une liste dite négative excluant de son champ d'application certains domaines, notamment les mines et les hydrocarbures, banques, assurances et réassurances, production d'armement et activités connexes militaires, ainsi que la production d'explosifs etc. La législation congolaise en matière d'assurance est constituée de textes disparates et obsolètes, outre qu'elle demeure encore en marge des instruments internationaux ${ }^{6}$. Conformément à l'article 202 point 36 de la Constitution, il est donc nécessaire de mettre sur pied une législation uniforme, moderne et complète, sous forme d'un code des assurances prenant en compte tous les engagements internationaux en matière d'assurances ainsi que les particularités du pays.

Précisons que depuis l'adoption de la loi n¹5/005 du 17 mars 2015 portant code des assurances, l'État congolais a libéralisé ce secteur mettant ainsi fin au monopole de la Société nationale d'assurances. À ce jour, le gouvernement congolais a pris le décret $\mathrm{n}^{\circ}$ 16/001 du 26 janvier 2016 portant création, organisation et fonctionnement de l'autorité de régulation et de contrôle des assurances.Le décret du 26 janvier 2016, détermine les modalités d'application de la loi $\mathrm{n}^{\circ} 15 / 005$ du 17 mars 2015 portant code des assurances et crée en même temps l'Autorité de régulation et de contrôle des assurances. L'article $4 \mathrm{du}$ décret cité ci-haut énonce que l'ARCA est un organe de régulation et de contrôle du secteur des assurances. Elle dispose de tous les pouvoirs qui lui sont nécessaires en vue d'exercer les actions prévues par le code des assurances. De même, elle veille à la protection des droits des assurés et des bénéficiaires des contrats d'assurances, à la solidité de l'assise financière des entreprises d'assurances et de réassurance ainsi qu'à leur capacité à honorer leurs engagements (article 4). L'ARCA a le pouvoir d'agréer les entreprises d'assurance et

4 RDC : 1'ANAPI propose la réduction du coût de création d'entreprise, disponible sur https://www.radiookapi.net/2017/10/03/actualite/economie/rdc-lanapi-propose-la-reduction-ducout-de-creation-dentreprise, 2017.

5 Lire notamment le décret-loi du 30 août 1965, l'ordonnance-loi n69/032 du 26 juin 1969, 1'ordonnance-loi n79/027 du 27 septembre 1979 et l'ordonnance-loi n86/033 du 12 mars 1986.

6 Raymond Ntungu, Le droit congolais à l'épreuve de la mobilité du capital : Protection de l'État récepteur d'investissements, Paris, L'Harmattan, 2013, p. 14. 
de réassurance ainsi que leurs dirigeants. C'est dans ce contexte, que l'ARCA a pu accorder le 28 mars 2019, soit 4 ans après sa création les premiers agréments à quatre sociétés privées d'assurance, parmi lesquelles les filiales de Rawbank Rawsur SA et Rwsur Life SA, et à deux sociétés de courtage afin qu'elles exercent leurs activités dans le secteur d'assurance, et ce de manière licite en $\mathrm{RDC}^{7}$.

La législation congolaise en matière d'investissement paraît plus attractive aujourd'hui, parce que ce pays fait partie de ces États qui, vers les années 70, ont aveuglement appliqué, et ce, de manière rigoureuse, les politiques de nationalisation au nom du principe de la souveraineté territoriale de l'État sur les ressources naturelles.Ces politiques avaient créé, par voie de conséquence, un sentiment d'insécurité juridique et politique pour les opérateurs économiques installés sur le territoire congolais. Les très nombreuses guerres qu'a connues le Congo entre 1996 et 2003 ont terni également l'image de ce pays. La RDC est toujours donc considérée par les investisseurs étrangers comme un pays à haut risque dans lequel il est difficile d'entreprendre une activité économique.En l'absence d'une vraie politique publique en matière d'investissement, il s'est avéré nécessaire pour les nouvelles autorités de trouver des solutions à même d'aider le législateur congolais à prendre de nouvelles mesures pour redonner confiance aux investisseurs étrangers. C'est dans ce contexte qu'un certain nombre de codes ont été adoptés par le parlement de transition issu des négociations politiques de Sun City en Afrique du Sud signée le 19 avril 2002 mais sans mandat du peuple congolais. Ces instruments juridiques avaient été demandés par la Banque Mondiale, et avaient permis aux entreprises multinationales d'investir dans les secteurs clé du pays entraînant un problème de concurrence déloyale entre les entreprises étrangères et locales, alors que le Congo n'était pas préparé à intégrer dans son système juridique une économie de plus en plus mondialisée. Cette politique d'ouverture aux capitaux, certes louable pour l'État congolais, a été malheureusement mise en œuvre dans la précipitation, renforçant ainsi davantage l'impuissance d'un système juridique éclaté voire très hétéroclite ${ }^{8}$. Il apparaît ainsi que le droit applicable, « ... c'est-à-dire le droit formel des investissements est contrarié dans les faits. Ce qui explique les propositions pour une amélioration de la qualité de la gouvernance et du climat des affaires. Paradoxalement, ce droit formel semble être plus favorable aux investisseurs qu'à l'État récepteur d'investissement ».L'affirmation d'un régime incitatif et stratégique d'admission en RDC découle de plusieurs instruments juridiques applicables distinctement dans chaque domaine d'investissements concerné. Il s'agit notamment de la loi $\mathrm{n}^{\circ} 004 / 2002 /$ du 21 février 2002 portant code des investissements, de la loi du 9 mars 2018 -loi n¹8/001 modifiant et complétant la loi $n^{\circ} 007 / 2002$ du 11 juillet 2002 portant Code minier, de la loi $n^{\circ} 11 / 2002$ du 29 août 2002 portant code forestier. Cela revient à dire que le code des investissements n'est pas le seul mécanisme juridique régissant l'établissement des investisseurs.

7 Bas Zuidberg, Les défis liés aux investissements dans les PMEs en RDC, Elan RDC, Kinshasa, Mars 2021, p.8.

8 Patrick Ndungidi, Investissement : la RDC rejoint l'Africa finance corporation, ADIC, juin 2021. 


\section{Bibliographie}

\section{A. Textes juridiques}

Constitution du 18 février 2006 telle que modifiée en 2011.

Décret $n^{\circ}$ 09/33 du 08 août 2009 portant statuts, organisation et fonctionnement de l'Agence pour la Promotion des Investissements, en sigle « ANAPI».

Loi nº04 du 21 février 2002 portant Code des investissements en RDC.

Décret du 30 juillet 1888, Les Codes Larcier, République Démocratique du Congo, Vol. 1 (2003)

\section{B. Ouvrage}

Kodo M.J, L'Application des actes uniformes de l'Ohada, Academia-Bruylant, 2011.

Mukola Kanuilo, La protection des investissements en droit international, Kinshasa, 2009.

\section{Articles et autres documents}

$R D C:$ l'ANAPI propose la réduction du coût de création d'entreprise, disponible sur https://www.radio okapi.net/2017/10/03/actualite/economie/rdc-lanapi-propose-la-reduction-du-cout-de-creation-dent reprise, 2017.

Raymond Ntungu, Le droit congolais à l'épreuve de la mobilité du capital : Protection de l'État récepteur d'investissements, Paris, L'Harmattan, 2013.

Bas Zuidberg, Les défis liés aux investissements dans les PMEs en RDC, Elan RDC, Kinshasa, Mars 2021, p.8.

Patrick Ndungidi, Investissement : la RDC rejoint l'Africa finance corporation, ADIC, juin 2021 